\title{
Design and Performance Analysis of a TLET-Type Flexure Hinge
}

\author{
Ngoc Le Chau, ${ }^{1}$ Ngoc Thoai Tran, ${ }^{1}$ and Thanh-Phong Dao $\mathbb{D}^{2,3}$ \\ ${ }^{1}$ Faculty of Mechanical Engineering, Industrial University of Ho Chi Minh City, Ho Chi Minh City, Vietnam \\ ${ }^{2}$ Division of Computational Mechatronics, Institute for Computational Science, Ton Duc Thang University, \\ Ho Chi Minh City, Vietnam \\ ${ }^{3}$ Faculty of Electrical \& Electronics Engineering, Ton Duc Thang University, Ho Chi Minh City, Vietnam
}

Correspondence should be addressed to Thanh-Phong Dao; daothanhphong@tdtu.edu.vn

Received 2 January 2020; Revised 16 March 2020; Accepted 23 March 2020; Published 9 April 2020

Academic Editor: Georgios I. Giannopoulos

Copyright (c) 2020 Ngoc Le Chau et al. This is an open access article distributed under the Creative Commons Attribution License, which permits unrestricted use, distribution, and reproduction in any medium, provided the original work is properly cited.

In order to permit a large deflection, three lamina emergent torsional flexure hinges are reconfigured to create a new triple LETtype flexure hinge (TLET) in this paper. The TLET consists of flexure hinges in a series coupled with others in parallel configuration. This arrangement is aimed to enhance the displacement of the joint. The proposed joint is capable of generating a large displacement and a large capacity of load within safety working conditions. The closed-form models are derived to calculate the equivalent spring constant, rotation angle, and displacement of the proposed joint. Failure analysis of the TLET joint with different materials is conducted by finite element analysis. The closed-form models are validated by simulations and experimentations. The validated results are well coincided each other. The result found that the joint achieves a maximum large displacement of $16.97 \mathrm{~mm}$ in the $x$-axis with respect to a maximum load of $20 \mathrm{~N}$. When the joint slides a maximum displacement of $16.97 \mathrm{~mm}$ along the $x$-axis, the output displacement emerges out the $z$-axis up to $23.12 \mathrm{~mm}$, respectively. The joint can achieve an angle displacement of $38.92^{\circ}$. The displacement of the TLET joint is 2.4 times greater than that of the traditional LET joint. The proposed joint is considered for engineering applications where a large working stroke and a large capacity of load are expected.

\section{Introduction}

Laminar emergent mechanism (LEM) is a subbranch of compliant mechanisms (CMs). LEM's functions are similar those of CMs which transfer force, motion, and energy [1-6]. CMs can be manufactured by the $3 \mathrm{D}$ printing technique, wire discharged machining process, or CNC machine from a solid $[7,8]$. Unlike CMs, LEM is fabricated from a sheet material with plastic or metal lamina material [9]. CM's motions are often in a fabrication plane while LEM's motion is emerged out the fabrication plane. When compared with traditional rigid-body mechanisms, LEMs take benefits of $\mathrm{CMs}$, e.g., monolithic and simple fabrication process, compact size, lightweight, reduced assemble components, free friction, no lubricant, and reduced wear [10-14]. LEM is considered as a mechanism where its functions are similar to a transmission mechanism, e.g., four bar mechanism, spherical LEMs $[15,16]$, slider crank mechanism [17, 18], and bistable collapsible compliant mechanism [19]. Due to the aforementioned advantages, the LEMs have been widely utilized for a variety of engineering applications, e.g., automobile industry, electronic industry, biomedical engineering, rescue equipment, micro-electromechanical system, cellphone and tablet holder, card syringe, lift table, multilayer shoes, solar panel, and membrane switch [18].

Over the past decade, the difficult challenge in designing LEMs is how to design a flexure hinge with a large deflection. For engineering applications in plane and space, a welldesigned flexure hinge should not provide a large deflection or a large rotating angle but it should ensure without plastic failures. In order to solve this situation, several researches focused on developing new shapes of flexure hinges. A basic research of membrane-enhanced LEMs for surrogate folds was developed [20], and LEM's joints were recommended for credit card [21]. In the light of resolving the LEM's challenges, RUFF and TUFF flexure hinges were investigated [22]. To expand the family of lamina emergent torsional (LET) joints, researchers developed LET joints with a large 
displacement such as ODLET [23], LET array for circuit board product [24], and spatial LET joints [25, 26]. In order to analyze behaviors of a LEM's flexure hinges, several methods have been developed, including the closed-form model [24], compliance matrix method [25, 27, 28], semianalytic model [29], finite element method [30], and pseudorigid-body model [31]. Besides, researches proposed advanced analytical methods to model a nonlinear relation of load versus displacement, e.g., analytical models for spatial compliant parallel modules [32], a simplified pseudo-rigidbody model method for spatial multibeam modules [33], position- and space-based design of a symmetric spatial compliant mechanism [34], nonlinear kinetostatic modelling for parallel manipulator [35], and normalization-based approach [36]. These techniques are still effective in solving a large deflection of flexure hinges. In the present article, a closed-form model based on the beam theory is adopted to analyze the performances of the TLET joint.

It is noted that a LET joint has a very thin-thickness in a fabrication plane and can result in a plastic deformation failure, fatigue damage, creep, and buckling phenomenon. Therefore, designing and analyzing for those behaviors of LET joint are critical task. With a fast development of an advanced human-machine interaction, robot end-effectors desire a large working stroke, a large rotation angle, a simple structure, and a safety operation. In order to meet such demands, a new class of LET joint is proposed in this article. The joint can permit a large displacement, wide capacity of load, and safety working condition. The joint has a potential application for the developed stage for deployable mechanisms.

The new contributions of this paper are to design and analyze a new triple LET joint (TLET) that is capable of allowing a large range of stroke and wide range of loads with no failures. The structure of proposed joint is constructed by connecting three LET hinges in an array by coupling them in series and parallel configuration. Subsequently, this work formulates closed-form models so as to calculate the equivalent spring constant. Based on the established equations, force-displacement curve and torque-angle displacement curve are described. The failure analyses of the triple LET joint are conducted through different materials. The closed-form models are then validated by finite element analysis and experiments. Finally, behaviors and performances of the TLET joint are compared with those of a conventional LET joint.

\section{Design of the TLET-Type Flexure Hinge}

In order to make a large displacement range when subjecting loads, this study proposes a new LET array, named TLET joint, as demonstrated in Figure 1. Generally, a number of LETs may be designed with two ones; however, when a LET with large displacement is required, the number of LETs should be raised. An array of coupled LETs is varied to change the displacement or stiffness. In this study, the TLET joint includes three coupled LETs is chosen to investigate the performances of an array of LETs. Based on a LET array, the proposed joint achieves a large displacement in the $x$-axis

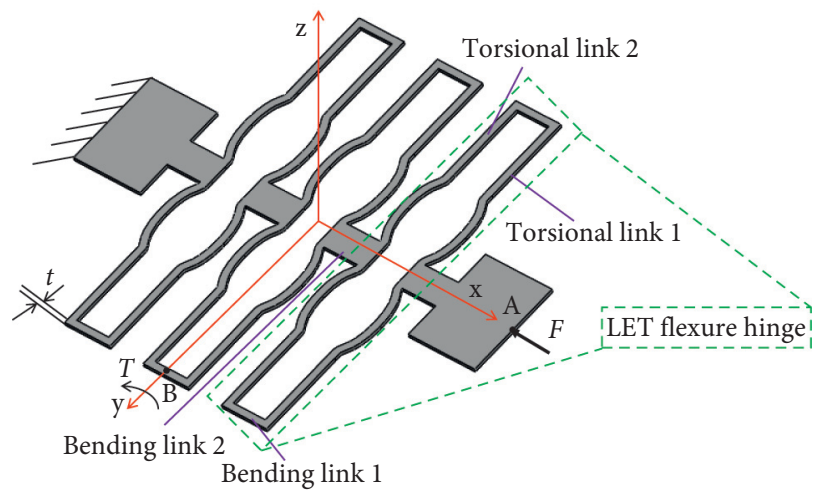

Figure 1: 3D model of the TLET joint.

(in-of-fabrication plane), a wide range of load, a large displacement in the $z$-axis (out-of-fabrication plane), and a large rotation angle around the $y$-axis. Under a torque $T$ around the $y$-axis, the TLET-type flexure hinge can subject a torsional deformation and a rotating deformation, simultaneously. TLET joint consists of torsional links 1, 2 and bending links 1,2, respectively. By taking advantages of such series and parallel arrangement, a stroke along the $x$-axis and an angular displacement around the $y$-axis are enhanced. The proposed hinge includes thickness $t$, width $w$, length $l$, and other geometrical parameters of torsional links and bending links, as shown in Figures 1 and 2. Table 1 gives main geometrical parameters of the joint. Summarily, the joint is proposed in terms of a large capacity of loads, a large displacement, and a large angular displacement without plastic deformation and buckling phenomenon failure.

As illustrated in Figure 2, the TLET joint has a symmetrical configuration about the horizontal and vertical directions (red dash lines). The lengths of torsional link 1 and torsional link 2 are $l_{T 1}$ and $l_{T 2}$, respectively. These dimensions are equal to each other. The width of two torsional links are named as $w_{T 1}$ and $w_{T 2}$, respectively. Meanwhile, the lengths of two bending links 1 and 2 are assigned as $l_{B 1}$ and $l_{B 2}$, respectively. The width of bending links is $w_{B 1}$ and $w_{B 2}$, respectively. The following list is the details of design parameters.

$$
\begin{aligned}
& l_{T 1} \text { : length of torsional link } 1 \\
& l_{T 2}: \text { length of torsional link } 2 \\
& l_{B 1} \text { : length of two bending link } 1 \\
& l_{B 2} \text { : length of two bending link } 2 \\
& w_{B 1} \text { : width of bending link } 1 \\
& w_{B 2}: \text { width of bending link } 2 \\
& w_{T 1}: \text { width of torsional link } 1 \\
& w_{T 2}: \text { width of torsional link } 2 \\
& w \text { : width of the TLET joint } \\
& t: \text { thickness of the TLET joint } \\
& l: \text { length of the TLET joint }
\end{aligned}
$$

Boundary conditions are included as follows: compression load, $F$, is applied at point $\mathrm{A}$ along the $x$-axis to cause a compressive displacement ( $x$-displacement) and an 


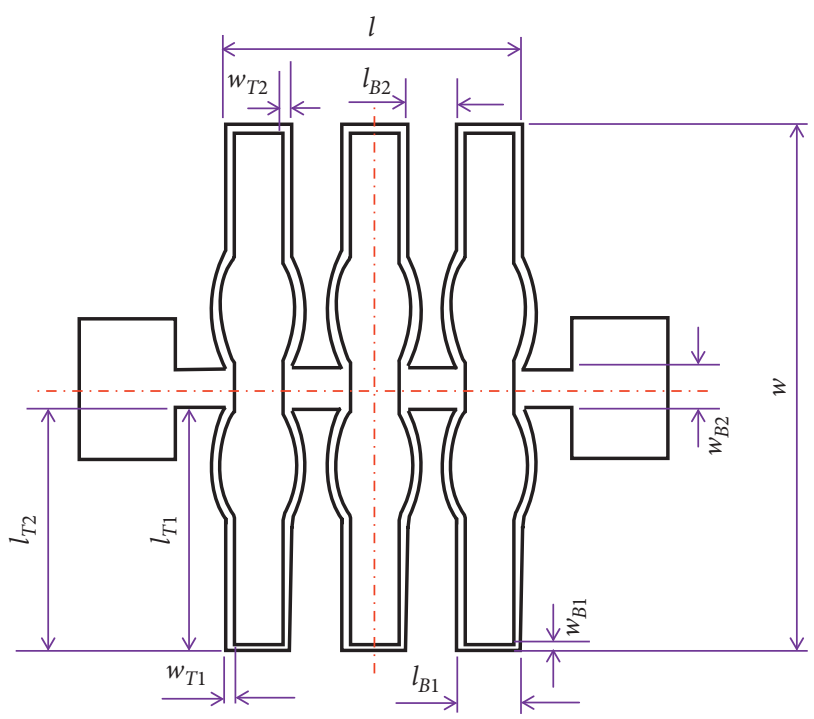

FIgUre 2: Geometrical dimensions of the TLET joint.

TABLe 1: Dimension of the TLET-type flexure hinge (unit: $\mathrm{mm}$ ).

\begin{tabular}{lccccccccccc}
\hline Symbol & $l_{T 1}$ & $l_{T 2}$ & $l_{B 1}$ & $l_{B 2}$ & $w_{B 1}$ & $w_{B 2}$ & $w_{T 1}$ & $w_{T 2}$ & $w$ & $t$ & $l$ \\
\hline Value & 49 & 49 & 14 & 10 & 2 & 8 & 2 & 2 & 107 & 1 & 60 \\
\hline
\end{tabular}

out-of-plan emerged displacement ( $z$-displacement). Torsion load, $T$, which is used to achieve the out-of-plane motion, is exerted a moment about the longitudinal axis of a torsional segment (the $y$-axis), at point B.

\section{Closed-Form Modelling}

In order to analyze the behaviors and performances of the TLET joint, the equivalent equations of spring constants are determined. Each of bending links and torsional links can be simplified as the bending and torsional springs because each segment is assumed as an elastic spring. Therefore, a schematic diagram of the stiffness of proposed TLET joint is assumed to be equivalent to elastic springs in series and parallel connections, as depicted in Figure 3. Whole stiffness of TLET joint can be classified into the one of spring's systems I, II, and III, where the bending link 2 is connected among these three systems.

Based on the connections in Figure 3, the equivalent stiffness system of TLET joint is described, as in Figure 4.

Based on the diagram in Figure 3, a set of springs, including $k_{T 1}, k_{B 1}$ and $k_{T 2}$, are arranged in a series, and then they are arranged in a parallel spring system, labeled I. Considering a top side of the system I which includes three springs $K_{T 1}, K_{B 1}$, and $K_{T 2}$ arranged in series, their equivalent stiffness is determined as follows:

$$
k_{\text {eq-topside-I }}=\frac{k_{T 1} k_{B 1} k_{T 2}}{k_{B 1} k_{T 2}+k_{T 1} k_{T 2}+k_{B 1} k_{T 1}},
$$

where $k_{\text {eq-topside-I }}, k_{T 1}, k_{B 1}$, and $k_{t 2}$ represent the equivalent stiffness of topside of spring's system I, stiffness of torsional link 1, stiffness of bending link 1 , and stiffness of torsional link 2, respectively.

As given in Figure 3, the bottom side of the system I is similar to the top side of this system. Therefore, total equivalent stiffness of the spring's system I is computed as

$$
K_{\mathrm{eqI}}=2 \frac{k_{T 1} k_{B 1} k_{T 2}}{k_{B 1} k_{T 2}+k_{T 1} k_{T 2}+k_{B 1} k_{T 1}}
$$

where $K_{\text {eqI }}$ depicts total equivalent stiffness of the spring's system I.

In a similar way, the equivalent stiffness of a top side of spring's system II is determined by

$$
k_{\text {eq-topside-II }}=\frac{k_{T 2} k_{B 1}}{2 k_{B 1}+k_{T 2}},
$$

where $k_{\text {eq-topside-II }}, k_{T 2}$, and $k_{B 1}$ are the equivalent stiffness of topside of spring's system II, stiffness of torsional link 2 , and stiffness of bending link 1 , respectively.

As a whole, the equivalent stiffness of the spring's system II is computed as

$$
K_{\text {eqII }}=2 \frac{k_{T 2} k_{B 1}}{2 k_{B 1}+k_{T 2}},
$$

where $K_{\text {eqII }}$ depicts total equivalent stiffness of the spring's system I.

According to Figure 3, total equivalent stiffness of the spring's system III is equal to the equivalent stiffness of spring's system I, which is determined by

$$
K_{\text {eqIII }}=2 \frac{k_{T 1} k_{B 1} k_{T 2}}{k_{B 1} k_{T 2}+k_{T 1} k_{T 2}+k_{B 1} k_{T 1}},
$$

where $K_{\text {eqIII }}$ represents the total equivalent stiffness of the spring's system III.

As shown in Figure 4, all springs are arranged in series and parallel connections. Based on this assumption, the equivalent spring constant, $K_{\text {eq-TLET, of the TLET-type }}$ flexure hinge is described as

$$
\frac{1}{K_{\text {eq-TLET }}}=\frac{1}{K_{\text {eqI }}}+\frac{1}{k_{B 2}}+\frac{1}{K_{\text {eqII }}}+\frac{1}{k_{B 2}}+\frac{1}{K_{\text {eqIII }}} .
$$

Hence, total equivalent spring constant of whole TLETtype joint is determined by

$$
K_{\text {eq-TLET }}=\frac{K_{\text {eqI }} K_{\text {eqII }} k_{B 2}}{2 k_{B 2} K_{\text {eqII }}+2 K_{\text {eqI }} K_{\text {eqII }}+K_{\text {eqI }} k_{B 2}},
$$

where the stiffness of bending element 1 , bending link 1 , and bending link 2 are calculated by

$$
\begin{aligned}
& k_{B 1}=\frac{E w_{B 1} t^{3}}{12 L_{B 1}}, \\
& k_{B 2}=\frac{E w_{B 2} t^{3}}{12 L_{B 2}},
\end{aligned}
$$

where $E$ is Young's modulus.

The stiffnesses of torsional link $1\left(k_{T 1}\right)$ and torsional link $2\left(k_{T 2}\right)$ are computed by 


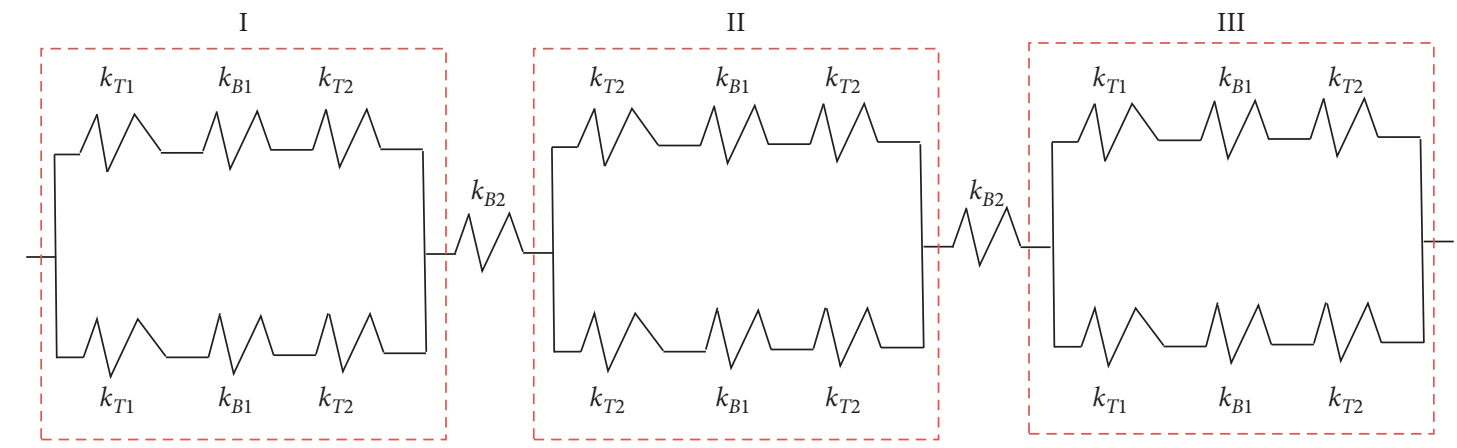

Figure 3: Schematic diagram of stiffness of the TLET-type flexure hinge.

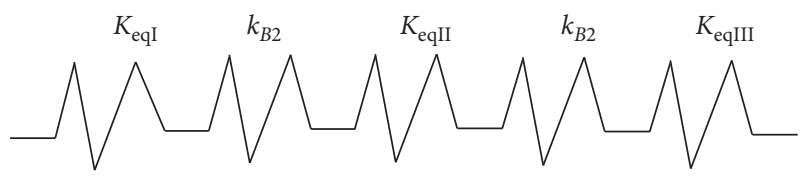

FIgURE 4: Schematic diagram of equivalent stiffness of the TLETtype flexure hinge.

$$
\begin{aligned}
& k_{T 1}=w_{T 1} t^{3} \frac{G}{L_{T 1}}\left[\frac{1}{3}-0.21 \frac{t}{w_{T 1}}\left(1-\frac{t^{4}}{12 w_{T 1}^{4}}\right)\right], \\
& k_{T 2}=w_{T 2} t^{3} \frac{G}{L_{T 2}}\left[\frac{1}{3}-0.21 \frac{t}{w_{T 2}}\left(1-\frac{t^{4}}{12 w_{T 2}^{4}}\right)\right],
\end{aligned}
$$

where

$$
G=\frac{E}{2(1+v)},
$$

where $G$ is the modulus of rigidity and $v$ is Poisson's ratio.

A relation between the input load in the $x$-axis versus the corresponding output displacement of the TLET joint is computed by

$$
\Delta=\frac{F}{K_{\text {eq-TLET }}},
$$

where $F$ is the load and $\Delta$ represents the displacement of the TLET joint along the $x$-axis.

By subjecting a pure torque around the $y$-axis, the rotation angle of the TLET joint is determined by

$$
\theta=\frac{T}{K_{\text {eq-TLET }}},
$$

where $\theta$ represents the rotation angle of the TLET-type flexure hinge.

\section{Analysis of Failures}

During the working process of the TLET joint, obstacles/ restrictions appear such as damage, creep, buckling, plastic, and fracture. These are undesired failures. Among aforementioned failures, the plastic deformation failure and the buckling phenomenon are the two most important failures which need to be resolved for the TLET hinge. The proposed joint can safely work within an elastic limitation of the material. Therefore, this section features various types of failures (plastic deformation and buckling phenomenon) so as to guarantee a safety working operation for the TLET joint.

4.1. Plastic Deformation Failure. The TLET hinge can fulfill the practical application if it works under the elastic area of the specific material. The purpose of the failure analysis is to guarantee a reliable working condition for the joint. Hence, stress limit and range of deformation of the joint are considered in this section. As known, if the resulting stress of the TLET joint is far higher than the yield strength of proposed material, the TLET joint is failed with plastic deformation failure. Therefore, selecting a proper material in terms of a large displacement and a large range of loads are the critical aspects, which are considered in this paper.

In order to explore this analysis, behavior and performance of the TLET joint which were made by structural steel, stainless steel, acrylonitrile butadiene styrene (ABS), polyethylene, and AL T73-7075 were compared with each other. The finite element method was adopted before fabricating a physical prototype. A 3-D model was created, and the finite element analysis was implemented by ANSYS 18.1 software. The face method was adopted for meshing the model. A nonlinear condition was applied for this analysis. Solid185 element was chosen, and the model was refined so as to guarantee a convergent result, as depicted in Figure 5. The results retrieved that the number of nodes is 38063 and the number of elements is 17559 . In order to ensure stability and accurate result of the simulations, an extra refinement for the meshed model was carried out at each three LET hinges. The results showed that the meshing quality is relatively good because average Skewness value is 0.48741 . The mechanical properties of each material for the simulations were given, as in Table 2. The failure analysis for each material was aimed to identify the maximum load, maximum stress limitation, and maximum displacement of the TLET joint. In all cases, the von Mises stress was considered. The boundary conditions and load, $F_{x}$, along the $x$-axis were setup, as depicted in Figure 5.

Made with structural steel, the TLET hinge could bear a maximum load of $11 \mathrm{~N}$ along the $x$-axis. Under this load, the TLET hinge could move a maximum displacement of $3.34 \mathrm{~mm}$ along the $x$-axis and a maximum von Mises stress 


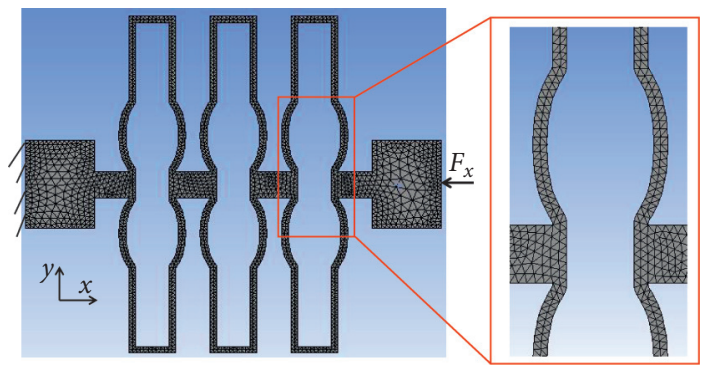

Figure 5: The meshed model of the TLET joint.

TABLE 2: Failure analysis of the TLET joint with various materials.

\begin{tabular}{|c|c|c|c|c|c|c|}
\hline Material & $\begin{array}{l}\text { Yield strength } \sigma_{y} \\
(\mathrm{MPa})\end{array}$ & $\begin{array}{l}\text { Young's modulus } \\
(\mathrm{MPa})\end{array}$ & $\begin{array}{l}\text { Max. load } \\
(\mathrm{N})\end{array}$ & $\begin{array}{l}\text { Max, stress } \sigma \\
(\mathrm{MPa})\end{array}$ & $\begin{array}{c}\text { Displacement } \\
(\mathrm{mm})\end{array}$ & Comparison \\
\hline Structural steel & 250 & 200000 & 11 & 236.54 & 3.34 & $\sigma_{y}>\sigma$ \\
\hline Stainless steel & 207 & 193000 & 9 & 193.27 & 2.83 & $\sigma_{y}>\sigma$ \\
\hline ABS & 43.6 & 2300 & 2 & 42.40 & 52.84 & $\sigma_{y}>\sigma$ \\
\hline Polyethylene & 25 & 1100 & 1 & 21.12 & 55.22 & $\sigma_{y}>\sigma$ \\
\hline Al T73-7075 & 503 & 71700 & 20 & 428.36 & 16.97 & $\sigma_{y}>\sigma$ \\
\hline
\end{tabular}

of $236.54 \mathrm{MPa}$ which was less than the yield strength of the structural steel $(250 \mathrm{MPa})$. Using the stainless steel, the TLET joint could bear a maximum load of $9 \mathrm{~N}$ and achieve a maximum displacement of $2.83 \mathrm{~mm}$ along the $x$-axis and a maximum stress of $193.57 \mathrm{MPa}$. This value of stress was far less than the yield strength of the stainless steel (207 MPa), as given in Table 2. By using the ABS material, the proposed joint could subject a maximum load of $2 \mathrm{~N}$, a maximum displacement of $52.84 \mathrm{~mm}$, and a maximum stress of 42.4 MPa which was still lower than the yield strength of ABS $(43.6 \mathrm{MPa})$. And then, using polyethylene, the proposed TLET could bear a maximum load of $1 \mathrm{~N}$ and move a maximum displacement of $55.22 \mathrm{~mm}$. The maximum stress was about of $21.12 \mathrm{MPa}$ which was smaller than the yield strength of polyethylene $(25 \mathrm{MPa})$. The results found that the above-used materials guarantee a reliable and safety work conditions for the TLET joint. Although the TLET joint allows a large working stroke, it only bears a maximum load up to $11 \mathrm{~N}$, as given in Table 2 . However, this study simultaneously considered the behaviors in terms of a large bearing capacity of load and a large working stroke for the proposed TLET joint. Subsequently, AL T73-7075 would be an alternative material to be considered for the TLET joint.

Made with AL T73-7075, the displacement of the TELT joint was $16.97 \mathrm{~mm}$ along the $x$-axis when a load of $20 \mathrm{~N}$ acted at the free end. The resulting von Mises stress was about $428.36 \mathrm{MPa}$. This stress was far lower than the yield strength of AL T73-7075 (503 MPa), as given in Table 2. This ensures a reliable work condition for the TLET hinge without plastic deformation failure. Then, an increase of the load's value was up to $25 \mathrm{~N}$, the TLET hinge could move a displacement of $21.21 \mathrm{~mm}$ but the resulting stress is about $535.45 \mathrm{MPa}$ which was far greater than the yield strength of AL T73-7075. At the load value of $25 \mathrm{~N}$ and above, the TLET joint failed to ensure reliability. Hence, the TLET joint with AL T73-7075 only ensures reliability with a maximum load of $20 \mathrm{~N}$ without failure. Through the aforementioned discussion, Al T73-7075 was chosen to fabricate a physical model of the TLET joint by wire electrical discharge machining (WEDM), as depicted in Figure 6(a). The mechanical properties of Al T73-7075 are given in Table 3 . Figure 6(b) illustrates the deformation of the TLET-type flexure hinge. To sum up, the TLET could move a maximum stroke $16.97 \mathrm{~mm}$ along the $x$-axis.

4.2. Analysis of Buckling Behavior. The first two buckling modes are investigated in this section. Under the load, $F$, along the $x$-axis, the TLET joint is compressed a displacement. At the same time, the out-of-plan displacement in the $z$-axis occurs. The buckling phenomenon also appeared herein. The first two modes of buckling behavior are analyzed to retrieve the multiplier load. The first two multiplier loads are smallest among all buckling modes, and therefore these first loads are easy to cause the buckling. So, the first two models of the buckling behavior are considered to determine the first two multiplier loads in this study. For a cantilever column, critical buckling load, $P_{c}$, can be determined [37]:

$$
P_{c}=\frac{E I \pi^{2}}{4 l^{2}}
$$

where $E$ is Young's modulus, $I$ parameter notes second moment of area, and $l$ is the length of a joint. Besides, the equation for the critical buckling load can be simplified as

$$
P_{c}=\lambda \times P_{I},
$$

where $\lambda$ is the eigenvalue or multiplier load.

By reusing the maximum value of load for each material in Table 2, the TLET joint might be subjected a buckling behavior. Using the finite element analysis, the multiplier load, $\lambda$, for the TLET hinge with different materials was analyzed, as provided in Table 4 . The results of this table 


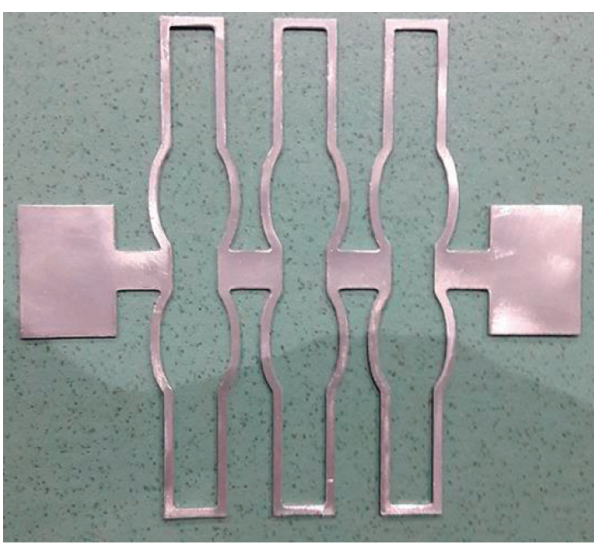

(a)

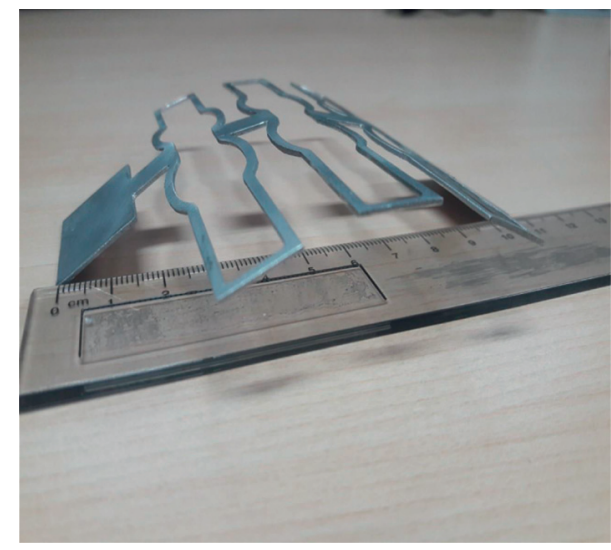

(b)

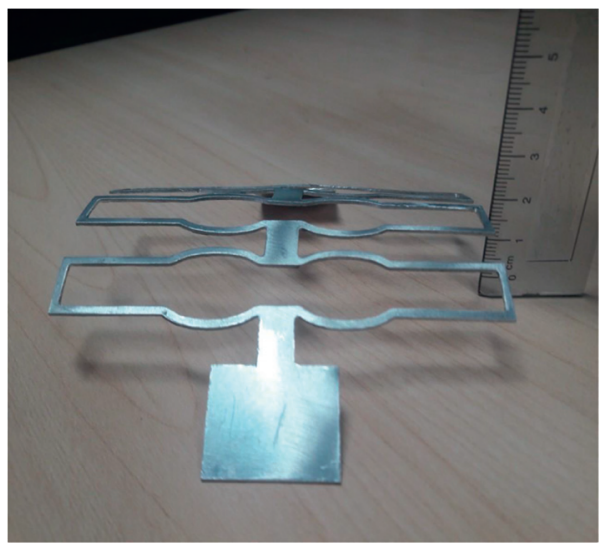

(c)

Figure 6: Physical model and deformation of the TLET joint: (a) prototype, (b) deformation in the $x$-axis, (c) deformation in the $z$-axis.

TABle 3: Mechanical properties of Al T73-7075.

\begin{tabular}{lccc}
\hline Young's modulus $(\mathrm{MPa})$ & Yield strength $(\mathrm{MPa})$ & Poisson ratio & Density $\left(\mathrm{kg} / \mathrm{m}^{3}\right)$ \\
\hline 71700 & 503 & 0.333 & 2770 \\
\hline
\end{tabular}

TABLE 4: Results of the buckling behavior for various materials.

\begin{tabular}{lccccc}
\hline Multiplier load & Structural steel & Stainless steel & ABS & Polyethylene & Al T73-7075 \\
\hline$\lambda$ of mode 1 & 0.34 & 0.40 & 0.02 & 0.02 & 0.06 \\
$\lambda$ of mode 2 & 2.64 & 3.09 & 0.15 & 0.15 & 0.51 \\
\hline
\end{tabular}

show that the values of multiplier load of the TELT with AL T73-7075 are lower than that of structural steel and stainless steel but larger than that of ABS and polyethylene, respectively. The results of the first two buckling modes for different materials are given in Table 4.

\section{Behavior Comparison of the TLET-Type Flexure Hinge and the Traditional LET}

The validity for the closed-form models would be validated by generating the simulations and experimentations in this part. The errors between the theory, simulation, and experiment were calculated.
The error between the theory and simulation was calculated by

$$
\varepsilon_{a}=\frac{\left|p_{\text {simu }}-p_{\text {theo }}\right|}{p_{\text {simu }}} * 100
$$

The error between the theory and experiment was computed by

$$
\varepsilon_{b}=\frac{\left|p_{\exp }-p_{\text {theo }}\right|}{p_{\exp }} * 100
$$

where $\varepsilon_{a}$ is the error between the theory and simulation, $\varepsilon_{b}$ is the error between theory and experiment, $P_{\text {simu }}$ is the 
TABLE 5: Comparison of displacement in the $x$-axis of TLET: theory, simulation, and experiment.

\begin{tabular}{|c|c|c|c|c|c|c|c|c|c|c|}
\hline Load $(\mathrm{N})$ & 2 & 4 & 6 & 8 & 10 & 12 & 14 & 16 & 18 & 20 \\
\hline$\Delta$-theory $(\mathrm{mm})$ & 1.63 & 3.27 & 4.91 & 6.54 & 8.18 & 9.82 & 11.46 & 13.10 & 14.74 & 16.38 \\
\hline$\Delta$-simulation $(\mathrm{mm})$ & 1.69 & 3.39 & 5.09 & 6.78 & 8.48 & 10.18 & 11.88 & 13.57 & 15.27 & 16.97 \\
\hline$\Delta$-experiment $(\mathrm{mm})$ & 1.55 & 3.11 & 4.67 & 6.22 & 7.78 & 9.34 & 10.90 & 12.45 & 14.01 & 15.57 \\
\hline$\varepsilon_{a}(\%)$ & 3.5 & & & & & & & & & \\
\hline$\varepsilon_{b}(\%)$ & 5.1 & & & & & & & & & \\
\hline
\end{tabular}

$\Delta$ represents the displacement along the $x$-axis

TABLE 6: Comparison of bending angle of TLET around $y$-axis: theory, simulation, and experiment.

\begin{tabular}{lcccccccccc}
\hline Torque $(\mathrm{N} \cdot \mathrm{mm})$ & 1 & 2 & 3 & 4 & 5 & 6 & 7 & 8 & 9 \\
\hline$\theta$-theory (degree) & 0.15 & 0.31 & 0.47 & 0.62 & 0.78 & 0.94 & 1.09 & 1.25 & 1.41 \\
$\theta$-simulation (degree) & 0.16 & 0.32 & 0.49 & 0.64 & 0.81 & 0.98 & 1.13 & 1.30 & 1.47 & 1.62 \\
$\varepsilon_{a}(\%)$ & 3.8 & & & & & & & & & \\
\hline
\end{tabular}

$\theta$ represents the angle displacement around the $y$-axis

simulation's value, $p_{\text {theo }}$ is the theoretical value, and $p_{\mathrm{ex}}$ is the experimental value.

In this study, the finite element analysis was implemented under the nonlinear conditions.

The results of Table 5 show that the error between the theory and simulation is around 3.5\%, while the error between the theory and experiment is about $5.1 \%$. These errors are caused from the approximately analytical model, error of meshing, error of finite element approximation, and error of fabrication and machine.

The rotation angle of the TLET hinge was recorded through simulations and theoretical calculations. Table 6 indicates that the errors between simulation and closedform model are approximately $3.8 \%$. It validates the correction of the established closed-form modelling. Figure 7 illustrates a coincidence between the simulations and theoretical results. By increasing the moment up to $248 \mathrm{~N} \cdot \mathrm{mm}$, the results found that the TLET hinge can rotate a maximum rotation angle of 38.92 degrees with a moment of $248 \mathrm{~N} \cdot \mathrm{mm}$ around the $y$-axis without failures.

In order to consider the highlight behavior and performances of the proposed TLET hinge, its behaviors would be compared with those of a traditional LET joint. The dimension of the LET joint includes length of $60 \mathrm{~mm}$ and height of $107 \mathrm{~mm}$. These dimensions are equal to the proposed TLET. Figures 8(a) and 8(b) give a CAD-model and a physical model of the traditional LET flexure hinge, respectively. The prototype of the LET joint was fabricated by the WEDM technique. A behavior comparison of the traditional LET with the proposed TLET would be analyzed, as shown in Figure 9.

Before implementing a comparison of the performances of the TLET versus the traditional LET joint, data are normalized. In this study, min-max normalization is used to scale the data between zero and unit. Normalizing equation is defined as

$$
z_{i}=\frac{x_{i}-x_{\min }}{x_{\max }-x_{\min }}
$$

where $z_{i}$ is the normalized data in range from 0 to 1 and $x_{i}$, $x_{\min }$, and $x_{\max }$ are ith data point, minimum value, and maximum value of data, respectively.

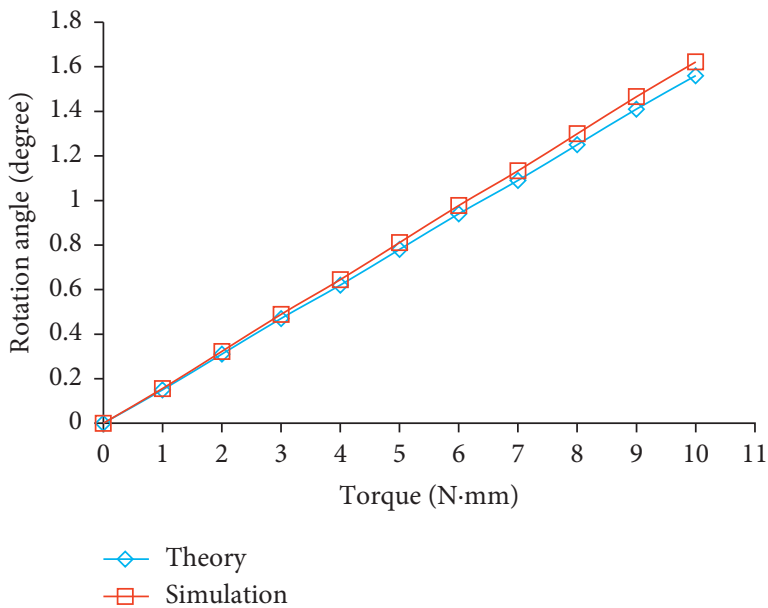

Figure 7: Comparison of the rotation angles between the theory and simulation.

Figure 9 depicts a comparison of the performances of the TLET hinge and the traditional LET flexure hinge. The results confirmed that the displacement of the TLET hinge is 2.47 times greater than that of the traditional LET hinge. The results found that performances of the designed flexure hinge are superior to those of the traditional one. If the thickness of the traditional one is reduced, its output displacement will be improved, but the output displacement of the proposed TLET is also better because the proposed flexure hinge is an array of three flexure hinges.

Considering the buckling phenomenon, the results found that the buckling multiplier (mode 1) of the TLET hinge is smaller than that of the traditional LET hinge, as given in Figure 10.

When the TLET subjects a compressive load along the $x$-axis, the TLET moves along the $x$-axis, and at the same time, it emerges out the $z$-axis. It means that a displacement point in the $x$-axis corresponds to that in the $z$-axis. Therefore, a relation between the input displacement of the TLET hinge along the $x$-axis and the output displacement along the $z$-axis (out-of-plane emerged motion) is described, as given in Figure 11. The result revealed that when 


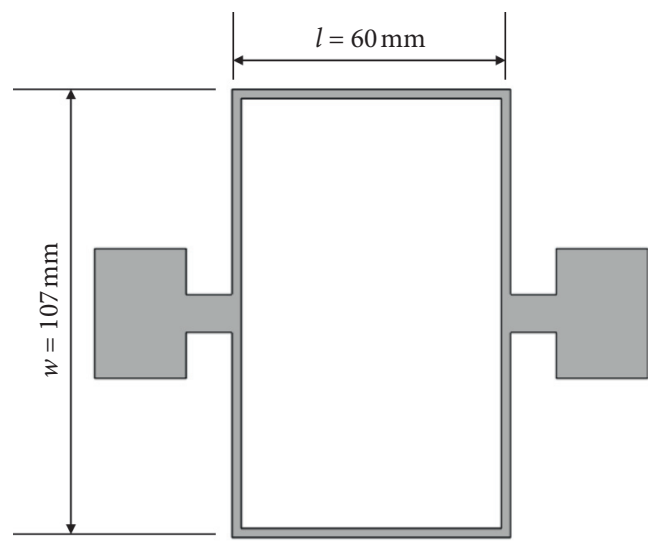

(a)

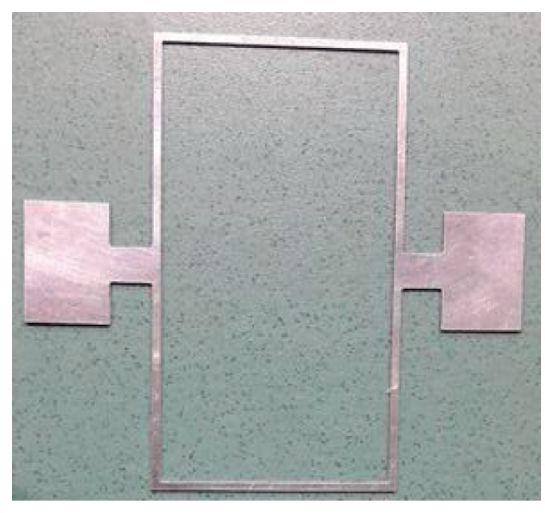

(b)

Figure 8: A traditional LET: (a) CAD model; (b) physical model.

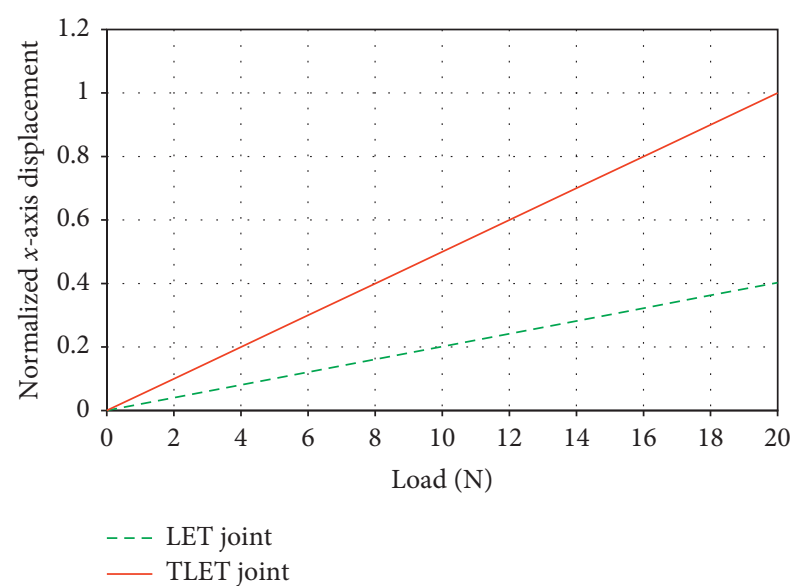

Figure 9: Comparison of the displacement between the LET joint and the TLET joint.

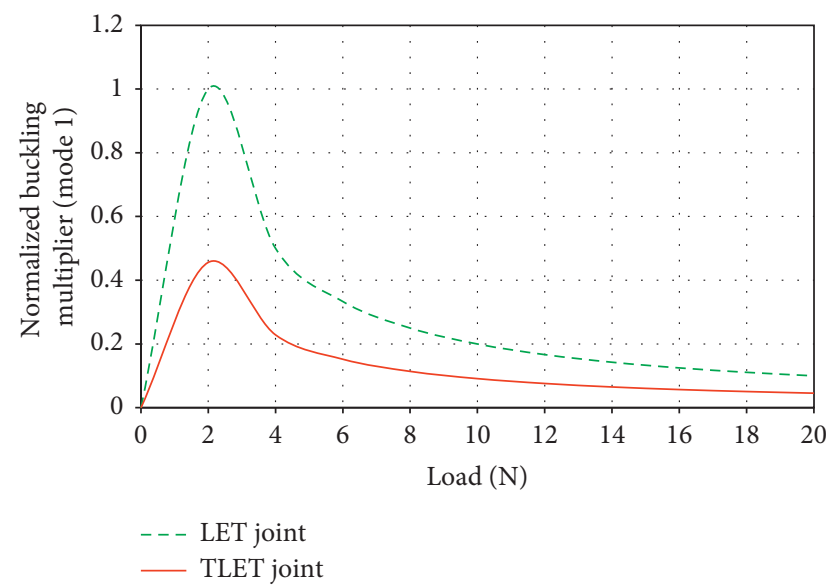

FIGURE 10: Comparison of the buckling between the LET joint and the TLET joint.

the TLET hinge can move a range from $1.69 \mathrm{~mm}$ to $16.97 \mathrm{~mm}$ along the $x$-axis, while the output emerged displacement of the TLET out the $z$-axis from $5.00 \mathrm{~mm}$ to

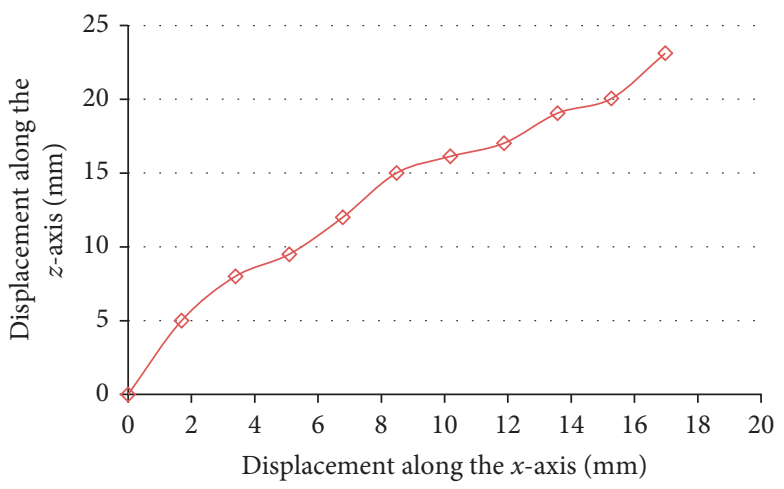

FIGURE 11: Relation between the displacement of $x$ - and $z$ - axes of the TLET joint.

$23.12 \mathrm{~mm}$, respectively. To sum up, the TLET-type flexure hinge can achieve a large working stroke of $16.97 \mathrm{~mm}$ along the $x$-axis and a large stroke of $23.12 \mathrm{~mm}$ along the $z$-axis, respectively. The behaviors of the proposed TLET hinge are far greater than those of the traditional LET hinge. The proposed joint with a LET array would be a proper candidate where there are large deformation and good capacity of load.

\section{Conclusions}

This paper proposed a new flexure hinge, the triple lamina emergent torsional joint. By reconfiguring three LET flexure hinges in an array of series and parallel configuration, the proposed TLET joint was able to permit a large displacement, a large range of load bearing capacity, and safety working condition without failures. The closed-form models for expressing the equivalent spring constant of the TLET hinge were deduced. By using the established equations, the force-displacement curve was illustrated and the rotation angle of the TLET joint was calculated. The overall stiffness was modeled via the analytical approach. Then, finite element analysis was used to verify the behavior of the joint in terms of deformation, maximum stress, and buckling instability for different materials. 
Since the TLET hinge only works in an elastic limitation of specific material, the analysis of failures for the joint was discussed. Several regarded materials for the TLET joint, such as structural steel, stainless steel, ABS, polyethylene, and Al T73-7075, were investigated and compared. The plastic deformation failure and buckling behavior of each case were analyzed. Al T73-7075 was chosen for the TLET joint. While the input displacement of $16.97 \mathrm{~mm}$ acted to the free end of the TLET along the $x$-axis, the joint could achieve a large deformation over $23.12 \mathrm{~mm}$ where its motion was emergent out the out-of-fabrication plane. In terms of a large displacement range and wide range of load, the TLET joint was capable of gaining the maximum displacement of $23.12 \mathrm{~mm}$ in the $z$-axis, the maximum displacement of $16.97 \mathrm{~mm}$ in the $x$-axis, and a wide capacity of load up to $20 \mathrm{~N}$ without failures. The results show that the TLET joint can achieve rotation angle of 38.92 degrees around the $y$-axis without failures. Compared with the simulations and experimental results, the results of validation indicated that the error between theory and simulation is around 4\%; meanwhile, the error between theory and experiment is about $5 \%$. These results were in well agreement with each other. It means that the closed-form models were valid so as to express the equivalent stiffness of the TLET flexure. In comparison with a traditional LET joint, the results found that the displacement of the TLET joint is 2.47 times greater than that of the traditional LET hinge. The TLET joint can be utilized for engineering applications where a large working stroke and a wide range of load are desired.

Future research topic will focus on analyzing a center sift of TLET, and an extra number of physical experiments are conducted to validate the theoretical models and simulations.

\section{Data Availability}

The data used to support the findings of this study are included within the article.

\section{Conflicts of Interest}

The authors declare that they have no conflicts of interest.

\section{Acknowledgments}

This research is funded by the Vietnam National Foundation for Science and Technology Development (NAFOSTED) under grant no. 107.01-2019.14.

\section{References}

[1] J. O. Jacobsen, G. Chen, L. L. Howell, and S. P. Magleby, "Lamina emergent torsional (LET) joint," Mechanism and Machine Theory, vol. 44, no. 11, pp. 2098-2109, 2009.

[2] N. L. Ho, T. P. Dao, N. Le Chau, and S. C. Huang, "Multiobjective optimization design of a compliant microgripper based on hybrid teaching learning-based optimization algorithm," Microsystem Technologies, vol. 25, no. 5, pp. 20672083, 2018.
[3] D. N. Nguyen, N. L. Ho, T.-P. Dao, and N. L. Chau, "Multiobjective optimization design for a sand crab-inspired compliant microgripper," Microsystem Technologies, vol. 25, no. 10, pp. 3991-4009, 2019.

[4] K.-B. Choi, "Dynamics of a compliant mechanism based on flexure hinges," Proceedings of the Institution of Mechanical Engineers, Part C: Journal of Mechanical Engineering Science, vol. 219, no. 2, pp. 225-235, 2005.

[5] K.-B. Choi and C. S. Han, "Optimal design of a compliant mechanism with circular notch flexure hinges," Proceedings of the Institution of Mechanical Engineers, Part C: Journal of Mechanical Engineering Science, vol. 221, no. 3, pp. 385-392, 2007.

[6] J. Zhang, H.-W. Guo, and Z.-N. Zhang, "Design of flexure revolute joint based on double compliant curved beam," Proceedings of the Institution of Mechanical Engineers, Part C: Journal of Mechanical Engineering Science, vol. 233, no. 13, pp. 4521-4530, 2019.

[7] M. Ling, J. Cao, M. Zeng, J. Lin, and D. J. Inman, "Enhanced mathematical modeling of the displacement amplification ratio for piezoelectric compliant mechanisms," Smart Materials and Structures, vol. 25, no. 7, pp. 1-11, 2016.

[8] W.-L. Zhu, Z. Zhu, P. Guo, and B.-F. Ju, "A novel hybrid actuation mechanism based XY nanopositioning stage with totally decoupled kinematics," Mechanical Systems and Signal Processing, vol. 99, pp. 747-759, 2018.

[9] P. S. Gollnick, E. E. Savage, and L. L. Howell, Detc2011-48350 a Preliminary Study of Actuation Approaches for Lamina Emergent, American Society of Mechanical Engineers (ASME), New York, NY, USA, 2011.

[10] J. O. Jacobsen, Fundamental Components for Lamina Emergent Mechanisms, MSc thesis, Brigham Young University, Provo, Utah, 2008.

[11] T. P. Dao and S. C. Huang, "Design and analysis of a compliant micro - positioning platform with embedded strain gauges and viscoelastic damper," Microsystem Technologies, vol. 23, no. 2, pp. 441-456, 2016.

[12] N. Le Chau, H. G. Le, T. Dao, M. P. Dang, and V. A. Dang, "Efficient hybrid method of FEA-based RSM and PSO algorithm for multi-objective optimization design for a compliant rotary joint for upper limb assistive device," 2019, https://www.springer.com/engineering/electronics/j.

[13] N. Le Chau, T. P. Dao, and V. T. T. Nguyen, "Optimal design of a dragonfly-inspired compliant joint for camera positioning system of nanoindentation tester based on a hybrid integration of jaya-ANFIS," Mathematical Problems in Engineering, vol. 2018, Article ID 8546095, 16 pages, 2018.

[14] M. Ling, S. Chen, Q. Li, and G. Tian, "Dynamic stiffness matrix for free vibration analysis of flexure hinges based on non-uniform Timoshenko beam," Journal of Sound and Vibration, vol. 437, pp. 40-52, 2018.

[15] S. E. Wilding, L. L. Howell, and S. P. Magleby, "Spherical lamina emergent mechanisms," Mechanism and Machine Theory, vol. 49, pp. 187-197, 2012.

[16] H. C. Greenberg, Using Lamina Emergent Mechanisms to Address Needs for a Space Environment, Brigham Young University, Provo, UT, USA, 2011.

[17] J. O. Jacobsen, L. L. Howell, and S. P. Magleby, "Components for the design of lamina emergent mechanisms," in Proceedings of the 2007 ASME International Mechanical Engineering Congress and Exposition, Seattle, WA, USA, 2007.

[18] P. S. Gollnick, S. P. Magleby, and L. L. Howell, "An introduction to multilayer lamina emergent mechanisms," Journal of Mechanical Design, vol. 133, no. 8, 2011. 
[19] R. Alfattani and C. Lusk, "A lamina-emergent frustum using a bistable collapsible compliant mechanism," Journal of Mechanical Design, vol. 140, no. 12, pp. 1-9, 2018.

[20] G. Chen, S. P. Magleby, and L. L. Howell, "Membrane-enhanced lamina emergent torsional joints for surrogate folds," Journal of Mechanical Design, vol. 140, no. 6, pp. 1-10, 2018.

[21] N. B. Albrechtsen and L. L. Howell, DETC2011-48420, American Society of Mechanical Engineers (ASME), New York, NY, USA, 2017.

[22] D. B. Ferrell, Y. F. Isaac, S. P. Magleby, and L. L. Howell, "Development of criteria for lamina emergent mechanism flexures with specific application to metals," Journal of $\mathrm{Me}$ chanical Design, vol. 133, no. 3, pp. 1-9, 2011.

[23] Z. Xie, L. Qiu, and D. Yang, "Design and analysis of outsidedeployed lamina emergent joint (OD-LEJ)," Mechanism and Machine Theory, vol. 114, pp. 111-124, 2017.

[24] B. P. DeFigueiredo, T. K. Zimmerman, B. D. Russell, and L. L. Howell, "Regional stiffness reduction using lamina emergent torsional joints for flexible printed circuit board design," Journal of Electronic Packaging, vol. 140, no. 4, 2018.

[25] H. Zhang, X. Zhang, and B. Zhu, "A novel flexural lamina emergent spatial joint," Mechanism and Machine Theory, vol. 142, Article ID 103582, 2019.

[26] N. B. Albrechtsen and L. L. Howell, DETC2010-28 Identifying Potential Applications for Lamina Emergent, American Society of Mechanical Engineers (ASME), New York, NY, USA, 2010.

[27] N. Wang, Z. Zhang, X. Zhang, and C. Cui, "Optimization of a 2DOF micro-positioning stage using corrugated flexure units," Mechanism and Machine Theory, vol. 121, pp. 683-696, 2018.

[28] G. Chen, Y. Ma, and J. Li, "A tensural displacement amplifier employing elliptic-arc flexure hinges," Sensors and Actuators A: Physica, vol. 247, pp. 315-307, 2016

[29] T. J. Teo, I.-M. Chen, G. Yang, and W. Lin, "A generic approximation model for analyzing large nonlinear deflection of beam-based flexure joints," Precision Engineering, vol. 34, no. 3, pp. 607-618, 2010.

[30] J. Dearden, C. Grames, J. Orr, B. D. Jensen, S. P. Magleby, and L. L. Howell, "Cylindrical cross-axis flexural pivots," Precision Engineering, vol. 51, pp. 604-613, 2018.

[31] T.-P. Dao, S.-C. Huang, and P. T. Thang, "Hybrid Taguchicuckoo search algorithm for optimization of a compliant focus positioning platform," Applied Soft Computing, vol. 57, pp. 526-538, 2017.

[32] G. Hao, X. Kong, and R. L. Reuben, "A nonlinear analysis of spatial compliant parallel modules: multi-beam modules," Mechanism and Machine Theory, vol. 46, no. 5, pp. 680-706, 2011.

[33] G. Hao, "Simplified PRBMs of spatial compliant multi-beam modules for planar motion," Mechanical Sciences, vol. 4, no. 2, pp. 311-318, 2013.

[34] H. Li and G. Hao, "Position-space based design of a symmetric spatial translational compliant mechanism for micro-/ nano-manipulation," Micromachines, vol. 9, no. 4, p. 189, 2018.

[35] G. Hao and J. Yu, "Design, modelling and analysis of a completely decoupled XY compliant parallel manipulator," Mechanism and Machine Theory, vol. 102, pp. 179-195, 2016.

[36] G. Hao and X. Kong, "A normalization based approach to the mobility analysis of spatial compliant multibeam modules," Mechanism and Machine Theory, vol. 59, 2013.

[37] T.-P. Dao and S.-C. Huang, "Compliant thin walled joint based on zygoptera nonlinear geometry," Journal of $\mathrm{Me}$ chanical Science and Technology, vol. 31, no. 3, pp. 1293-1303, 2017. 